\title{
Epidemiology of, and behavioural risk factors for, sexually transmitted human papillomavirus infection in men and women in Britain
}

\author{
Anne M Johnson, ${ }^{1}$ Catherine H Mercer, ${ }^{1}$ Simon Beddows, ${ }^{2}$ Natasha de Silva, ${ }^{2}$ \\ Sarika Desai, ${ }^{2}$ Rebecca Howell-Jones, ${ }^{2}$ Caroline Carder, ${ }^{3}$ Pam Sonnenberg, ${ }^{1}$ \\ Kevin A Fenton, ${ }^{1}$ Catherine Lowndes, ${ }^{2}$ Kate Soldan ${ }^{2}$
}

\begin{abstract}
- An additional table is published online only. To view this file please visit the journal online (http://sti.bmj.com/ content/88/3.toc).

${ }^{1}$ Research Department of Infection and Population Health, University College London, Mortimer Market Centre, London, UK

${ }^{2}$ Health Protection Agency, London, UK

${ }^{3}$ Clinical Microbiology, University College Hospitals NHS Foundation Trust, The Windeyer Institute of Medical Sciences, London, UK
\end{abstract}

\section{Correspondence to} Dr Catherine H Mercer, Research Department of Infection and Population Health, University College London, Mortimer Market Centre, off Capper Street, London WC1E 6JB, UK; c.mercer@ucl.ac.uk

The data in this manuscript were presented as an oral presentation at the 19th meeting of the International Society for Sexually Transmitted Disease Research (ISSTDR) held in Québec City, Canada, 10-13 July 2011 (http://www. isstdrquebec2011.com).

Accepted 4 December 2011 Published Online First 17 January 2012

\begin{abstract}
Objectives Persistent infection with high-risk sexually transmitted human papillomaviruses (HR-HPVs) can lead to development of cervical and other cancers, while lowrisk types (low-risk HPV) may cause genital warts. We explored the epidemiology of different HPV types in men and women and their association with demographic and behavioural variables.
\end{abstract}

Methods We analysed data collected for the British National Survey of Sexual Attitudes and Lifestyles, a cross-sectional survey undertaken in 1999-2001. Half of all sexually experienced male and female respondents aged 18-44 years were invited to provide a urine sample. We tested 3123 stored urine samples using an in-house Luminex-based HPV genotyping system.

Results HPV DNA was detected in $29.0 \% 195 \% \mathrm{Cl}$ $26.7 \%$ to $31.3 \%$ ) of samples from women and $17.4 \%$ (95\% Cl $15.1 \%$ to $19.8 \%$ ) from men. Any of 13 HR-HPV types was detected in $15.9 \%$ (95\% Cl $14.1 \%$ to $17.8 \%)$ of women and $9.6 \%(95 \% \mathrm{Cl} 8.0 \%$ to $11.6 \%)$ of men. HPV types $16 / 18$ were found in $5.5 \%(95 \% \mathrm{Cl} 4.5 \%$ to $6.8 \%)$ of women and $3.0 \%(95 \% \mathrm{Cl} 2.1 \%$ to $4.3 \%)$ of men; and types $6 / 11$ in $4.7 \%(95 \% \mathrm{Cl} 1.8 \%$ to $5.9 \%)$ of women and $2.2 \%(95 \% \mathrm{Cl} 1.5 \%$ to $3.1 \%)$ of men. In multivariate analysis, HR-HPV was associated with new partner numbers, in women with younger age, single status and partner concurrency, and in men with number of partners without using condom(s) and age at first intercourse.

Conclusions HPV DNA was detectable in urine of a high proportion of the sexually active British population. In both genders, HR-HPV was strongly associated with risky sexual behaviour. The minority of HPV infections were of vaccine types. It is important to monitor HPV prevalence and type distribution following the introduction of vaccination of girls.

\section{INTRODUCTION}

Persistent infection with certain types of sexually transmitted human papillomavirus (HPV) can lead to the development of cervical and other cancers, while other types cause the majority of genital warts. HPV types that infect the genital area are categorised as 'high-risk' HPV (HR-HPV) if known to cause cervical cancer or 'low-risk' HPV (LR-HPV) if not, albeit an imperfect categorisation. ${ }^{12}$ Infection with HR-HPV types is necessary but not sufficient for development of cervical cancer. Thirteen types are currently recognised by the WHO International Agency for Research on Cancer as 'high risk'. ${ }^{3}$ HR-HPV is also implicated in the aetiology of some other anogenital cancers and of some oropharyngeal cancers. ${ }^{4}$ Types 16 and 18 are associated with $>70 \%$ of cervical cancers worldwide. ${ }^{5}$ Infection with HPV types 6 or 11 is associated with the majority of cases of anogenital warts. ${ }^{6}$

Genital infection is often asymptomatic and undiagnosed. However, genital warts remain the most commonly diagnosed viral sexually transmitted infection in the UK, with around 91000 cases diagnosed in genitourinary medicine clinics in 2009 , an increase of $30 \%$ since $2000 .^{7}$ Transmission is driven by the infectivity of the organisms, sexual behaviour and the effectiveness of control interventions. Once infected, it is estimated that $70 \%$ of women clear infection within 3 years. ${ }^{8}$ Surveillance of genital warts, detection of abnormalities by cervical screening and cancer registrations give some measures of the impact of HPV infection but do not show the population prevalence or distribution of HPV types.

An HPV immunisation programme was introduced in the UK in 2008 for 12-13-year-old girls, with a catch-up programme to age 18. ${ }^{9} 10$ The programme, to protect against cervical cancer, uses the bivalent vaccine protecting against types 16 and 18, but not types 6 and 11, which the quadrivalent vaccine offers.

Nationally representative epidemiological studies are needed to inform HPV immunisation policy and cervical cancer screening strategies and efforts to improve uptake. The frequency of HR-HPV types not present in current vaccines is an important baseline against which to monitor any indirect effect of vaccination on non-vaccine types (eg, type replacement or cross-protection). Here, we examine the proportion of men and women with detectable HPV infection in urine samples archived from a national probability sample survey of sexual attitudes and lifestyles (Natsal-2) undertaken prior to the introduction of HPV vaccination in Britain and the relationship between infection and demographic and behavioural variables. ${ }^{11}$

\section{METHODS}

The second National Survey of Sexual Attitudes and Lifestyles (Natsal-2) is a stratified probability bmi.com/site/about/unlocked. xhtml 
sample survey of the population aged 16-44 years, resident in Britain. Eleven thousand one hundred and sixty-one people, of whom 6399 were women, were interviewed between May 1999 and February 2001. Details of the methodology are published elsewhere. ${ }^{11}$ Briefly, a sample of 40523 addresses was selected from the small-user Postcode Address File for Britain with a multistage probability cluster design, with oversampling in Greater London. Interviewers visited all selected addresses. One resident aged 16-44 years from every eligible household was invited after random selection to participate. Natsal-2 achieved a response rate of $65.4 \%$. Interviewers conducted interviews in respondents' homes. A face-to-face interview included questions on socio-demographics, health behaviours, and first sexual experience. Respondents who reported no sexual experience of any kind in the face-to-face interview and those aged 16 and 17 years with some heterosexual experience but no heterosexual intercourse or same-sex experience reported in screening questions were not given the subsequent computer-assisted selfinterview. This included questions on the number and gender of sexual partners and sexual practices.

Five thousand and twenty-six respondents at alternate sampled addresses, aged 18-44 years, who reported at least one partner with whom they had sexual intercourse, were invited to provide a urine sample for initial testing for genital Chlamydia trachomatis (chlamydia) as previously reported. ${ }^{12}$ Three thousand five hundred and sixty-nine (71\%) agreed and gave signed informed consent for chlamydia testing and storage for anonymous future testing, providing $10 \mathrm{ml}$ of urine in a plastic sterile container containing $1 \mathrm{mg}$ of boric acid. The interviewer posted the urine sample to a central laboratory. Following testing for chlamydia, samples were stored at $-80^{\circ} \mathrm{C}$.

\section{Ethical approval}

Natsal-2 obtained ethical approval from University College Hospital, North Thames Multicentre, and all local research ethics committees in Britain. Approval for HPV testing of the stored urine samples was obtained from St Mary's Research Ethics Committee (Ref: 07/O0403/9).

\section{Laboratory methods}

Three thousand four hundred and thirty-six urine samples stored at $-80^{\circ} \mathrm{C}$ were available. Three thousand one hundred and twenty-three samples were adequate for testing using an inhouse Luminex-based genotyping assay for detection of HPV types $6,11,16,18,26,31,33,35,39,45,51,52,53,56,58,59,66$, $68,70,73$ and $82 .{ }^{13}$ Each $1 \mathrm{ml}$ urine sample was subjected to centrifugation (13000 rpm for $20 \mathrm{~min}$ ) and the pellet suspended in $300 \mu \mathrm{l}$ phosphate buffered saline before samples were lysed (40 $\mu \mathrm{l}$ Qiagen Protease and $265 \mu \mathrm{l}$ Qiagen Buffer AL) and nucleic acids extracted on the BioRobot Universal platform (Qiagen; OIAamp ${ }^{\circledR}$ DNA Blood BioRobot ${ }^{\circledR}$ MDx kit, Qiagen Limited, Crawley, West Sussex, UK). Ten microlitres of the $100 \mu$ l eluted material was used for PCR amplification. HPV DNA $(10 \mu \mathrm{l})$ was amplified using the in-house single-round multiplex PCR. The assay incorporated a control PCR to assess specimen integrity and resolved type-specific infections using a genotyping assay based on the Bio-Plex ${ }^{\circledR}$ (Luminex xMAP ${ }^{\circledR}$, Bio-Rad Laboratories, Hemel Hempstead, Hertfordshire, UK) platform.

\section{Statistical methods}

We did all statistical analyses in Stata $(\mathrm{V} .10)^{14}$ accounting for stratification, clustering and weighting of the sample. We used weighted data to correct for unequal selection probabilities and to match the age/sex population profile. ${ }^{11}$ We examined the relationship between HPV detection and socio-demographic and behavioural factors and used multivariate analyses to identify the factors associated with HR-HPV types detection. We defined HR-HPV as types 16, 18, 31, 33, 35, 39, 45, 51, 52, 56, 58, 59 and 68 according to WHO International Agency for Research on Cancer definition. ${ }^{3}$ We used stepwise logistic regression to model factors identified as statistically significant at $p<0.10$ in the bivariate analyses. The statistical significance threshold for inclusion in the final multivariate model was $p<0.10$. To account for differential demographic and behavioural response patterns in willingness to provide a urine sample, the data were given an additional weight, calculated by the inverse of the modelpredicted probability of responding. ${ }^{12}$

\section{RESULTS}

Table 1 shows the prevalence of detectable HPV DNA by type in urine for men and women. Any HPV DNA was detected in $29.0 \%$ of samples from women and $17.4 \%$ from men. Any HRHPV was detected in $15.9 \%$ of women and $9.6 \%$ of men. The detected prevalence of either HR-HPV type in current vaccines (HPV types 16 and 18) was 5.5\% in samples from women and $3.0 \%$ from men, with $4.1 \%$ of women and $2.6 \%$ of men having these types only. $9.8 \%$ of women and $4.8 \%$ of men had one of the quadrivalent vaccine types $(6,11,16$ and 18$) .3 .4 \%$ of women and $2.6 \%$ of men had two or more HR-HPV types detectable.

Table 1 HPV DNA positivity by type and gender

\begin{tabular}{lll}
\hline & $\begin{array}{l}\text { Women } \\
\text { \% (95\% Cl) }\end{array}$ & $\begin{array}{l}\text { Men } \\
\text { \% (95\% Cl) }\end{array}$ \\
\hline $\begin{array}{l}\text { Denominator* } \\
\text { HPV type }\end{array}$ & 1525,1851 & 1545,1272 \\
Any HPV & $29.0(26.7$ to 31.3$)$ & $17.4(15.1$ to 19.8$)$ \\
Any high risk $\dagger$ & $15.9(14.1$ to 17.8$)$ & $9.6(8.0$ to 11.6$)$ \\
2+ high-risk typest & $3.4(2.6$ to 6.6$)$ & $2.6(1.3$ to 4.9$)$ \\
High-risk type(s) 16 and/or 18 & $5.5(4.5$ to 6.8$)$ & $3.0(2.1$ to 4.3$)$ \\
High-risk type(s) 16 and/or 18 only & $4.1(3.1$ to 5.2$)$ & $2.6(1.8$ to 3.9$)$ \\
Low-risk type(s) 6 and/or 11 & $4.7(3.8$ to 5.9$)$ & $2.2(1.5$ to 3.1$)$ \\
Types 6/11/16/18 & $9.8(8.4$ to 11.3$)$ & $4.8(3.7$ to 6.3$)$ \\
LR HPV-6 & $3.1(2.3$ to 4.2$)$ & $1.1(0.7$ to 1.9$)$ \\
LR HPV-11 & $2.4(1.8$ to 3.3$)$ & $1.2(0.7$ to 2.0$)$ \\
HR HPV-16 & $4.2(3.3$ to 5.3$)$ & $2.3(1.5$ to 3.5$)$ \\
HR HPV-18 & $1.4(0.9$ to 2.2$)$ & $0.7(0.3$ to 1.4$)$ \\
LR HPV-26 & $0.9(0.5$ to 1.6$)$ & $0.3(0.1$ to 1.0$)$ \\
HR HPV-31 & $0.6(0.3$ to 1.1$)$ & $0.3(0.1$ to 0.9$)$ \\
HR HPV-33 & $0.9(0.6$ to 1.5$)$ & $0.4(0.1$ to 0.9$)$ \\
HR HPV-35 & $1.0(0.6$ to 1.6$)$ & $1.0(0.5$ to 1.8$)$ \\
HR HPV-39 & $1.0(0.6$ to 1.6$)$ & $0.7(0.3$ to 1.5$)$ \\
HR HPV-45 & $0.7(0.4$ to 1.2$)$ & $0.8(0.4$ to 1.6$)$ \\
HR HPV-51 & $1.9(1.3$ to 2.7$)$ & $1.5(1.0$ to 2.4$)$ \\
HR HPV-52 & $2.3(1.7$ to 3.2$)$ & $1.1(0.6$ to 1.9$)$ \\
LR HPV-53 & $2.0(1.4$ to 2.8$)$ & $0.8(0.4$ to 1.5$)$ \\
HR HPV-56 & $1.4(0.9$ to 2.1$)$ & $0.9(0.5$ to 1.9$)$ \\
HR HPV-58 & $1.7(1.2$ to 2.4$)$ & $0.6(0.3$ to 1.2$)$ \\
HR HPV-59 & $1.0(0.5$ to 1.8$)$ & $0.2(<0.01$ to 0.6$)$ \\
LR HPV-66 & $0.9(0.5$ to 1.5$)$ & $0.6(0.3$ to 1.2$)$ \\
HR HPV-68 & $1.8(1.2$ to 2.7$)$ & $0.9(0.5$ to 1.7$)$ \\
LR HPV-70 & $1.0(0.6$ to 1.6$)$ & $0.4(0.1$ to 0.9$)$ \\
LR HPV-73 & $0.9(0.6$ to 1.5$)$ & $0.4(0.2$ to 1.0$)$ \\
LR HPV-82 & $<0.01(<0.01$ to 0.3$)$ & $<0.01(<0.01$ to 0.6$)$ \\
\hline
\end{tabular}

*Weighted, unweighted denominator defined as respondents in Natsal-2 providing a urin sample.

†Defined as HPV types 16, 18, 31, 33, 35, 39, 45, 51, 52, 56, 58, 59, 68.

HR HPV, high-risk human papillomavirus; LR HP, low-risk human papillomavirus 
Table 2 Results from a stepwise logistic regression* to identify the key socio-demographic characteristics, sexual behaviours and health-related factors $\dagger$ associated with high-risk human papillomavirus DNA positivity: women

\begin{tabular}{|c|c|c|c|c|}
\hline & $\%(95 \% \mathrm{Cl})$ & $\begin{array}{l}\text { Crude OR } \\
\text { (95\% CI) }\end{array}$ & $\begin{array}{l}\text { Adjusted OR } \\
\text { (95\% CI) }\end{array}$ & Denominator \\
\hline All & 15.9 (14.1 to 17.8$)$ & - & - & 1525,1851 \\
\hline Age at interview, years & & $\mathrm{p}<0.0001$ & $p=0.045$ & \\
\hline $30+$ & 11.3 (9.5 to 13.3 ) & 1.00 & 1.00 & 915,1146 \\
\hline$<30$ & 22.7 (19.4 to 26.4$)$ & $2.32(1.78$ to 3.02$)$ & 1.37 (1.01 to 1.85$)$ & 610,705 \\
\hline Marital status & & $\mathrm{p}<0.0001$ & $\mathrm{p}<0.0001$ & \\
\hline Married & 7.2 (5.6 to 9.2 ) & 1.00 & 1.00 & 741,769 \\
\hline Cohabiting & 20.7 (15.9 to 26.4$)$ & 3.37 (2.21 to 5.15$)$ & 2.73 (1.78 to 4.18$)$ & 280,295 \\
\hline Previous married & 21.8 (16.3 to 28.6 ) & 3.61 (2.26 to 5.77$)$ & 2.68 (1.69 to 4.25$)$ & 138,245 \\
\hline Single, never married & 27.5 (23.2 to 32.3$)$ & 4.91 (3.49 to 6.90$)$ & 3.00 (1.99 to 4.53$)$ & 366,542 \\
\hline $\begin{array}{l}\text { Number of new sexual } \\
\text { partners, } \S \text { last year }\end{array}$ & & $\mathrm{p}<0.0001$ & $p=0.0006$ & \\
\hline 0 & 11.3 (9.6 to 13.2 ) & 1.00 & 1.00 & 1174,1363 \\
\hline 1 & 29.1 (23.3 to 35.5$)$ & 3.22 (2.28 to 4.55$)$ & 1.55 (1.08 to 2.22 ) & 200,293 \\
\hline $2+$ & 42.3 (32.6 to 52.7 ) & 5.77 (3.72 to 8.94$)$ & 2.47 (1.53 to 3.99 ) & 98,138 \\
\hline $\begin{array}{l}\text { Concurrency between } \\
\text { most recent sexual } \\
\text { partners, } \S \text { ๆ last year }\end{array}$ & & $\mathrm{p}<0.0001$ & $\mathrm{p}=0.032$ & \\
\hline No & $13.9(12.2$ to 15.9$)$ & 1.00 & 1.00 & 1333,1599 \\
\hline Yes & 31.1 (23.6 to 39.7 ) & 2.78 (1.86 to 4.16$)$ & 1.59 (1.04 to 2.44$)$ & 101,144 \\
\hline
\end{tabular}

*Variables significant at $p<0.10$ in the bivariate analyses (supplemental table, available online) were considered in the stepwise logistic regression where the significance level for addition to the model is $p<0.10$. Note that the resulting model also applies when the significance level for addition is $p<0.05$. When the significance level for addition is $p<0.01$, there are only two variables in the model: marital status and the 'number of new opposite/same-sex partners, last year'. No pairwise interactions were statistically significant.

†In order to make the model more statistically efficient, response categories were collapsed where there were no significant differences between categories.

‡Weighted, unweighted denominator defined as respondents in Natsal-2 providing a urine sample. Respondents with data missing for $1+$ variable(s) were excluded from the multivariate analyses.

§opposite and/or same sex sexual partner(s).

ॠRespondents were asked to report the months and years of first and last sex with their most recent partners (at most three partners). Where these dates overlapped between their most recent partner and their second most recent partner and/or their second most recent partner and their third most recent partner, it is assumed that the partnerships were concurrent.

Further analyses examine the factors associated with HR-HPV types. In both women and men, prevalence of detectable HR-HPV declined with age from over one-quarter of women aged 18-24 years to less than a tenth of women aged 40-44 years (supplemental table, available online). HR-HPV was least frequent among those who were married but was not associated with either educational status or social class. Since over $90 \%$ of the sample was of white ethnicity, sample sizes for non-white groups were too small to permit robust analysis of ethnic variations.

In bivariate analysis, prevalence of HR-HPV was strongly associated with sexual behaviour in men and women (supplemental table, available online). Prevalence fell with rising age at first intercourse, more markedly for men than women. HR-HPV was strongly associated with increasing numbers of lifetime partners, and, in the last year, the total number and new partners, partners without a condom and partner concurrency (ie, where dates of sex overlapped between the most recent partner and the second most recent partner and/or the second most recent partner and the third most recent partner). As an example, prevalence rose from $11.3 \%$ of women with no new partner in the last year to $42.3 \%$ of those with two or more new partners in the last year (unadjusted OR 5.77 (95\% CI 3.72 to 8.94)). Reporting ever having a same-sex partner was associated with HR-HPV in women but not in men. Smoking and alcohol consumption were significantly associated with HR-HPV risk in men and women, as was oral contraception use in women. HR-HPV was less common in circumcised men, but this was of borderline statistical significance. Reporting previous STI diagnosis/es was significantly associated with HR-HPV in men only. Positivity for chlamydia was significantly associated with HR-HPV, with prevalence of $28.3 \%$ (95\% CI $13.6 \%$ to $49.9 \%$ ) and $9.2 \%$ (95\% CI $7.5 \%$ to $11.2 \%)$ in men with and without chlamydia infection, respectively, detected in their urine (unadjusted OR 3.91 (95\% CI $1.50 \%$ to $10.14 \%$ )) and $39.5 \%$ (95\% CI $24.4 \%$ to $56.9 \%$ ) and $15.4 \%$ (95\% CI $13.7 \%$ to $17.4 \%$ ) in women with and without chlamydia infection, respectively, (unadjusted OR 3.57 (95\% CI 1.74 to 7.34 )).

We undertook a stepwise regression analysis to identify the demographic and behavioural factors most strongly associated with HR-HPV. Table 2 shows that for women, in the final model, HR-HPV remained significantly associated with younger age, being unmarried, increasing numbers of new sexual partners in the last year and concurrency between the most recent sexual partners in the last year. For men (table 3), HR-HPV remained significantly associated with younger age at first sex, increasing numbers of sexual partners without a condom in the last year and the number of new sexual partners in the last year.

We also examined the relationship between receptive oral sex and HPV. Receptive oral sex in the last year was commonly reported by women (cunnilingus) $(77.9 \%, 95 \%$ CI $75.8 \%$ to $80.0 \%$ ) and by men (fellatio) $(79.0 \%, 95 \%$ CI $76.2 \%$ to $81.6 \%)$. HR-HPV DNA positivity was higher in those reporting receptive oral sex, in men only (supplemental table, available online). This indicates considerable potential for HPV transmission to oropharyngeal sites of partners. The prevalence of reporting receptive oral sex did not vary by age group among men but declined significantly among women aged 35-44 years. Heterosexual anal sex in the last year was reported by $12.7 \%$, (95\% CI $10.9 \%$ to $14.8 \%$ ) of men and $11.7 \%$, 
Table 3 Results from a stepwise logistic regression* to identify the key socio-demographic characteristics, sexual behaviours and health-related factors $†$ associated with human papillomavirus DNA positivity of high-risk types: men

\begin{tabular}{|c|c|c|c|c|}
\hline & $\%(95 \% \mathrm{Cl})$ & $\begin{array}{l}\text { Crude OR } \\
(95 \% \mathrm{CI})\end{array}$ & $\begin{array}{l}\text { Adjusted OR } \\
\text { (95\% CI) }\end{array}$ & Denominator $\neq$ \\
\hline All & 9.6 (8.0 to 11.6$)$ & - & - & 1545,1272 \\
\hline $\begin{array}{l}\text { Age at first heterosexual } \\
\text { sex, years }\end{array}$ & & $p=0.0001$ & $p=0.0199$ & \\
\hline $18+$ & 5.6 (3.6 to 8.5 ) & 1.00 & 1.00 & 489,417 \\
\hline 16 or 17 & 9.5 (6.8 to 13.0$)$ & $1.77(0.98$ to 3.23$)$ & $1.23(0.70$ to 2.14$)$ & 572,461 \\
\hline$<16$ & 15.7 (11.9 to 20.4$)$ & $3.16(1.86$ to 5.36$)$ & 2.06 (1.19 to 3.56$)$ & 429,345 \\
\hline $\begin{array}{l}\text { Number of sexual } \\
\text { partners§ without } \\
\text { a condom, last year }\end{array}$ & & $\mathrm{p}<0.0001$ & $p=0.0106$ & \\
\hline 0 & 3.6 (1.8 to 7.0$)$ & 1.00 & 1.00 & 319,301 \\
\hline 1 & 9.1 (7.1 to 11.5$)$ & 2.68 (1.25 to 5.77$)$ & 2.34 (1.13 to 4.89$)$ & 1001,752 \\
\hline $2+$ & 24.1 (17.0 to 33.1$)$ & 8.59 (3.71 to 19.9 ) & 3.59 (1.56 to 8.29$)$ & 187,186 \\
\hline $\begin{array}{l}\text { Number of new sexual } \\
\text { partners, } \S \text { last year }\end{array}$ & & $\mathrm{p}<0.0001$ & $p=0.0347$ & \\
\hline 0 & 6.7 (5.0 to 8.9$)$ & 1.00 & 1.00 & 1029,786 \\
\hline 1 & 12.7 (8.4 to 18.8 ) & 2.03 (1.14 to 3.61$)$ & 1.33 (0.73 to 2.43 ) & 240, 219 \\
\hline $2+$ & 22.6 (16.7 to 29.8 ) & 4.07 (2.51 to 6.59 ) & 2.35 (1.23 to 4.48$)$ & 216,220 \\
\hline
\end{tabular}

*Variables significant at $p<0.10$ in the bivariate analyses (supplemental table, available online) were considered in the stepwise logistic regression where the significance level for addition to the model is $p<0.10$. Note that the resulting model also applies when the significance level for addition is $p<0.05$. When the significance level for addition is $p<0.01$, the only variable remaining significant in the model is the 'number of opposite/same-sex partners without a condom, last year'. No pairwise interactions were statistically significant.

†In order to make the model more statistically efficient, response categories were collapsed where there were no significant differences between categories.

$\ddagger$ Weighted, unweighted denominator defined as respondents in Natsal-2 providing a urine sample. Respondents with data missing for $1+$ variable(s) were excluded from the multivariate analyses.

$\S 0$ pposite and/or same sex sexual partner(s).

(95\% CI $10.2 \%$ to $13.4 \%$ ) of women. Women (only) reporting this behaviour were more likely to be detectable HR-HPV DNA positive.

For completeness, we also carried out analysis of the relationship between HPV 16 and/or 18 and demographic and behavioural variables (data not shown). Given the lower prevalence, we had less power than for the analysis of any HR type, but this analysis was broadly consistent with that for all HR-HPV types (supplemental table, available online).

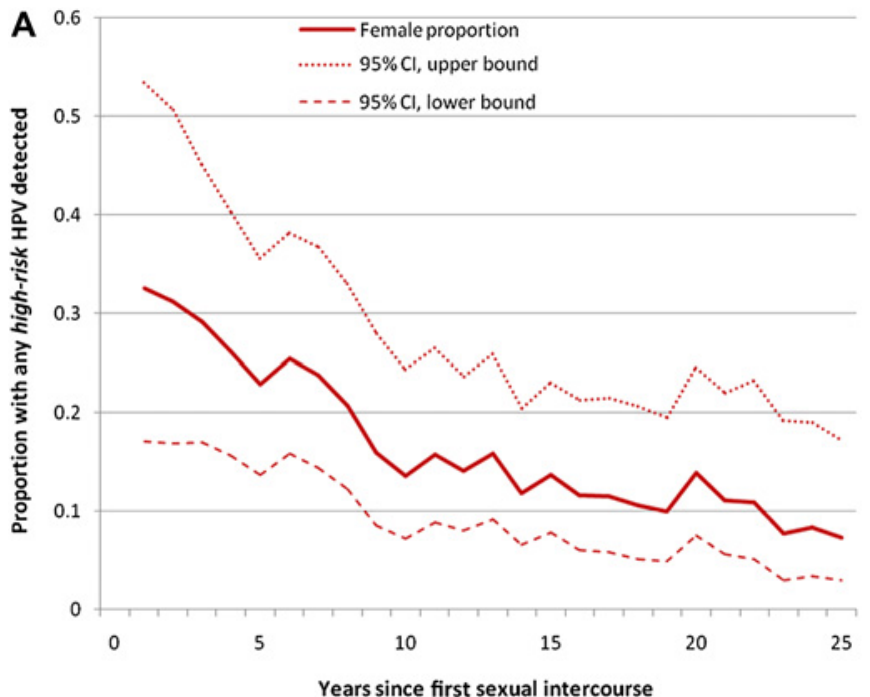

Of those found to have HPV 6 and/or 11, the types predominantly associated with genital warts, only $1.9 \%(95 \% \mathrm{CI}$ $0.6 \%$ to $6.2 \%$ ) of women and $4.8 \%$ (95\% CI $0.7 \%$ to $27.7 \%$ ) of men reported that they had been informed by a doctor that they had genital warts/HPV infection.

The median age at first intercourse was 17 for both women and men. In an analysis of the time since first intercourse (figure $1 \mathrm{~A}, \mathrm{~B})$, infection appears to be acquired in a high proportion of young women within a year of first intercourse, with a greater

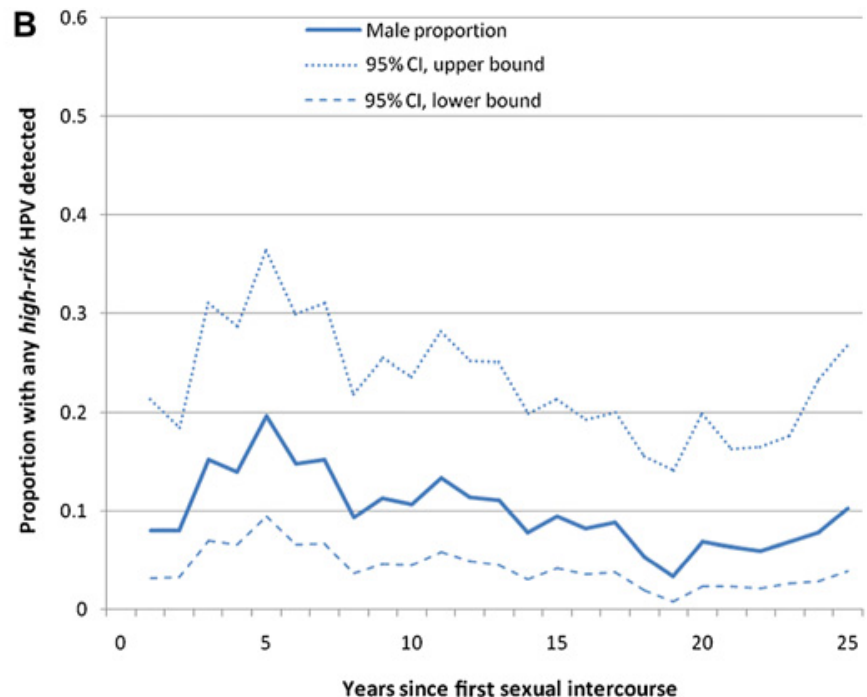

Figure 1 (A) Detection of any high-risk human papillomavirus (HPV) by years since first sexual intercourse: Females denominator: Sexually experienced respondents aged 18-44 years in Natsal-2, 3-point moving average. (B) Detection of any high-risk HPV by years since first sexual intercourse: Males denominator: Sexually experienced respondents aged 18-44 years in Natsal-2, 3-point moving average. This figure is produced in colour in the online journal-please visit the website to view the colour figure. 
lag among men. This may reflect gender differences in the age of first sexual partners, as women tend to have first intercourse with partners who are older and who are more likely to be sexually experienced. ${ }^{15}$ Alternatively, or additionally, this may reflect increased biological susceptibility to HPV infection of the immature transformation zone in young girls.

\section{DISCUSSION}

This is the first population-based probability sample study of the distribution of sexually transmissible HPV types in Britain with detailed analysis of the relationship with demographic and behavioural variables, and including men. We have shown that sexually transmissible HPV is highly prevalent in the population with HPV DNA detectable in $29 \%$ of urine samples from women and $17 \%$ of samples from men. However, of those infected, less than one-third had HPV 16/18.

The overall HPV prevalence is similar to that observed for women by the National Health and Nutrition Examination Survey (NHANES) study in the USA in 2003-2004, which used self-taken vaginal swabs (27\% in women aged $14-59$ years). However, prevalence of HPV 16 DNA was higher in Natsal-2 $(4.2 \%)$ than in NHANES (1.5\%), as was HPV 18 , at $1.4 \%$ in Natsal-2 and $0.8 \%$ in NHANES. ${ }^{16}$ This may in part be explained by the younger age of the Natsal-2 participants and the exclusion of participants with no sexual experience.

In common with other studies, ${ }^{17}$ we detected HPV DNA less frequently in samples from men than from women. This may reflect genuine difference in prevalence. However, urine is known to be an imperfect sample for detection of genital HPV infection. In validation studies comparing HPV detection in paired samples from urine and genital swabs from men and urine and liquid-based cytology samples from women, we have shown urine samples to have lower sensitivity, particularly in men, although specificity approaches $100 \%$, ${ }^{13}$ consistent with the work of others. ${ }^{16} 1819$ Taking account of the long period of storage of samples in this survey and the lower sensitivity of HPV assays in urine than in vaginal or urethral swabs, our results are likely to be minimum estimates. ${ }^{13}$

Men are key to the transmission of HPV to women, but relatively little is known about HPV infection in men. The sexual behaviour data in this population-based study allowed us to demonstrate, despite the reduced sensitivity of the HPV measure in men, which may lead to misclassification, that similar associations with behavioural characteristics are seen for men as women. The prevalence in men is lower than that in the recent paper by Giuliano et al, ${ }^{20}$ but participants in that study were drawn from a non-random sample in a number of countries and testing was based on genital swabs so a direct comparison is not possible here.

In NHANES, prevalence was highest in women aged 20-24 years and when the sexually inactive were excluded, and, as in Natsal-2, prevalence then declined with increasing age. ${ }^{16}$ Previous studies in England have shown that prevalence of HPV is highest among the youngest women attending for cervical screening. ${ }^{21}$ These studies have not explored socio-demographic and behavioural characteristics affecting these patterns. Declining HPV DNA prevalence with age and with time from first intercourse is consistent with reduced partner change with increasing age in Natsal-2, ${ }^{11}$ clearance of infection over time, with or without any naturally acquired immunity. ${ }^{8}$

Socio-demographic and behavioural data allowed us to explore the relationship between HPV and past and recent risk factors. The strong relationship between HPV risk and sexual behaviour was striking. In particular, we demonstrated the strong associa- tion with recent acquisition of new partners in both sexes consistent with HPV infections being transient. While in bivariate analysis, we are able to identify a range of health behavioural and demographic variables associated with presence of HPV, in the adjusted regression model, many of these behaviours no longer remained significant and are likely to have been confounders rather than on the causal pathway. The association with chlamydia infection (detected in urine) is also likely to be the result of confounding, as very similar risk factors have previously been shown for chlamydia infection. ${ }^{12}$ In our population, the minority of men were circumcised, but there was some evidence in bivariate analyses of a protective effect albeit of borderline statistical significance, while risk increased among those with multiple partners and no condom use. Numerous studies have shown associations between smoking, cervical cancer and HPV infection. $^{22}$ We have previously shown that those who have more partners are likely to smoke and drink more alcohol. ${ }^{23} 24$ In our study, the association between smoking and HPV infection no longer remained significant in multivariate analysis suggesting confounding of the association by sexual behaviour.

The observed increases in oropharyngeal squamous cell carcinomas in recent years seem to be attributable to increasing HR-HPV (HPV 16/18 in particular) and increasing practice of orogenital sex. ${ }^{25}$ In the first National Survey of Sexual Attitudes and Lifestyles undertaken in 1990-1991, we documented a considerable increase in orogenital contact through the latter half of the 20th century. ${ }^{23}$ Given the high prevalence of

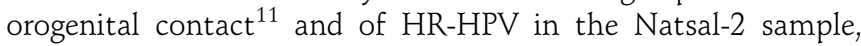
there is substantial potential for transmission of HR-HPV from genital to oral sites of infection.

The high proportion of young women with detectable HPV and the finding that many are infected within a year or two from first intercourse underscores the importance of delivering HPV vaccination programmes before girls become sexually active. Our analysis of HPV16/18 demonstrated similar risk factors to those for all HR types and supports the existing policy to offer HPV vaccination to all young women (younger than 18 years) in the UK and may help to inform decisions about vaccination of some older women. It is striking that despite the lower sensitivity of urine

\section{Key messages}

- Our data from a representative cross-sectional sample survey of the British population allow for the first time direct comparison between men and women and the risk factors for HPV acquisition.

- Sexually transmissible HPV is highly prevalent in the population with HPV DNA detectable in $29 \%$ of urine samples from women and $17 \%$ of samples from men. However, of those infected, less than one-third had HPV 16 or 18.

- There is a very strong relationship in both men and women between recent sexual partner change and HPV DNA and rapid acquisition in women after first intercourse. Condom use has some protective effect even after taking account of numbers of sexual partners.

- Our data provide evidence for the ongoing debate on the costeffectiveness of HPV vaccination in young men given the increasing number of cancers, which are thought to be related to HPV and the importance of continued sexual transmission of HPV when only women are vaccinated. 
samples in men for detecting HPV, it could be detected in a relatively high proportion. This finding provides evidence for the ongoing debate on the cost-effectiveness of vaccinating young men.

These estimates of sexually transmissible HPV frequency prior to introducing vaccination in girls provide a baseline to measure changes in vaccine- and non-vaccine-type HPV, in vaccinated women and unvaccinated women and in men who should benefit from 'herd immunity' effects due to reduced transmission of infection. HPV 16/18 vaccination has been shown to confer some cross-protection against some closely related non-vaccine HPV types, particularly HPV 31, 33 and $45^{26}$

HPV vaccination is an expensive public health intervention, and it will be important to examine whether vaccination of women reduces the prevalence of infection, in both genders. We are currently undertaking on a third National Survey of Sexual Attitudes and Lifestyles in which urine samples will be collected and self-reported vaccine uptake measured. Fieldwork is scheduled to be completed in 2012 and will allow comparative estimates between the two surveys a decade apart.

Funding The Natsal-2 study was funded by the UK Medical Research Council (grant number: G99811620) with funds from the Department of Health, the Scottish Executive and the National Assembly for Wales. HPV testing was funded by the Department of Health Policy Research Programme (grant number: 039/0030). The views expressed are not necessarily those of the Department of Health or Medical Research Council. The authors' work was independent of the funders, who had no role in the design, collection, analysis or interpretation of the data or the decision to submit for publication.

\section{Competing interests None.}

Patient consent Obtained.

Ethics approval Natsal-2 study obtained ethical approval from University College Hospital, North Thames Multicentre, and all local research ethics committees in Britain. Approval for HPV testing of the stored urine samples was obtained from St Mary's Research Ethics Committee (Ref: 07/00403/9).

Contributors AMJ and KAF were responsible for the study design of Natsal-2 and oversaw the collection of urine samples. CML, KS and AMJ were responsible for the study design and secured funding for the present study. SB and NdS were responsible for the HPV testing. RH-J and SD were responsible for data management at the HPA. $\mathrm{CHM}$ undertook all statistical analyses. All authors contributed to the design and interpretation of the data. AMJ wrote the first draft of the manuscript, but all authors contributed to revising the manuscript and approved the final version of the manuscript.

Provenance and peer review Not commissioned; externally peer reviewed.

\section{REFERENCES}

1. Muñoz N, Bosch FX, de Sanjose S, et al. Epidemiologic classification of human papillomavirus types associated with cervical cancer. N Engl J Med 2003;348:518-27.

2. Muñoz N, Castellsagué $X$, de González $A B$, et al. Chapter 1: HPV in the etiology of human cancer. Vaccine 2006;24(Suppl 3):S3/1-10.

3. Bouvard V, Baan R, Straif $K$, et al. A review of human carcinogens-Part B: biological agents. Lancet Oncol 2009;10:321-2.
4. Parkin DM, Bray F. Chapter 2: the burden of HPV-related cancers. Vaccine 2006;24 (Suppl 3):S3/11-25

5. Li N, Franceschi S, Howell-Jones R, et al. Human papillomavirus type distribution in 30,848 invasive cervical cancers worldwide: variation by geographical region, histological type and year of publication. Int J Cancer 2011;128:927-35.

6. Vandepapeliere $\mathbf{P}$, Barrasso R, Meijer CJ, et al. Randomized controlled trial of an adjuvanted human papillomavirus (HPV) type 6 L2E7 vaccine: infection of external anogenital warts with multiple HPV types and failure of therapeutic vaccination. $J$ Infect Dis 2005;192:2099-107.

7. Health Protection Agency. STI Annual Data Tables 2009. 2011. http://www.hpa. org.uk/stiannualdatatables/ (accessed 29 Nov 2011).

8. Moscicki AB, Shiboski S, Broering J, et al. The natural history of human papillomavirus infection as measured by repeated DNA testing in adolescent and young women. J Pediatr 1998;132:277-84.

9. Harper DM, Franco EL, Wheeler CM, et al. Sustained efficacy up to 4.5 years of a bivalent $L 1$ virus-like particle vaccine against human papillomavirus types 16 and 18: follow-up from a randomised control trial. Lancet 2006:367:1247-55.

10. Jit M, Choi YH, Edmunds WJ. Economic evaluation of human papillomavirus vaccination in the United Kingdom. BMJ 2008;337:a769.

11. Johnson AM, Mercer $\mathrm{CH}$, Erens B, et al. Sexual behaviour in Britain: partnerships, practices, and HIV risk behaviours. Lancet 2001;358:1835-42.

12. Fenton KA, Korovessis C, Johnson AM, et al. Sexual behaviour in Britain: reported sexually transmitted infections and prevalent genital Chlamydia trachomatis infection. Lancet 2001;358:1851-4.

13. Bissett SL, Howell-Jones R, Swift C, et al. Human papillomavirus genotype detection and viral load in paired genital and urine samples from both females and males. J Med Virol 2011;83:1744-51.

14. Stata Statistical Software: Release 10 [Computer Program]. College Station, TX, USA StataCorp LP, 2007

15. Mercer $\mathbf{C H}$, Wellings K, Macdowall W, et al. First sexual partnerships: age differences and their significance. Empirical evidence from the 2000 British National Survey of Sexual Attitudes and Lifestyles ('Natsal 2000'). J Adolesc Health 2006:39:87-95.

16. Dunne EF, Unger ER, Sternberg M, et al. Prevalence of HPV infection among females in the United States. JAMA 2007;297:813-19.

17. Dunne EF, Nielson CM, Stone KM, et al. Prevalence of HPV infection among men: a systematic review of the literature. J Infect Dis 2006;194:1044-57.

18. Weaver BA, Feng 0 , Holmes $\mathrm{KK}$, et al. Evaluation of genital sites and sampling techniques for detection of human papillomavirus DNA in men. $J$ Infect Dis 2004; 189:677-85

19. Daponte A, Pournaras S, Mademtzis I, et al. Evaluation of high-risk human papillomavirus types PCR detection in paired urine and cervical samples of women with abnormal cytology. J Clin Virol 2006;36:189-93

20. Giuliano AR, Lee JH, Fulp W, et al. Incidence and clearance of genital human papillomavirus infection in men (HIM): a cohort study. Lancet 2011;377:932-40. Erratum in: Lancet 2011;377:2006.

21. Howell-Jones R, Bailey A, Beddows $S$, et al. Multi-site study of HPV type-specific prevalence in women with cervical cancer, intraepithelial neoplasia and normal cytology, in England. Br J Cancer 2010;103:209-16.

22. Appleby $\mathbf{P}$, Beral V, Berrington de GA, et al. Carcinoma of the cervix and tobacco smoking: collaborative reanalysis of individual data on 13,541 women with carcinoma of the cervix and 23,017 women without carcinoma of the cervix from 23 epidemiological studies. Int J Cancer 2006;118:1481-95.

23. Johnson AM, Wadsworth J, Wellings K, et al. Sexual Attitudes and Lifestyles. Oxford: Blackwell Scientific Publications, 1994.

24. Aicken CR, Nardone A, Mercer $\mathrm{CH}$. Alcohol misuse, sexual risk behaviour and adverse sexual health outcomes: evidence from Britain's national probability sexual behaviour surveys. J Public Health (Oxf) 2010;33:262-71.

25. Mehanna H, Jones TM, Gregoire V, et al. Oropharyngeal carcinoma related to human papillomavirus. BMJ 2010;340:c1439.

26. Paavonen J, Naud P, Salmeron J, et al. Efficacy of human papillomavirus (HPV)-16/ 18 AS04-adjuvanted vaccine against cervical infection and precancer caused by oncogenic HPV types (PATRICIA): final analysis of a double-blind, randomised study in young women. Lancet 2009:374:301-14. 